\title{
Posterior occipitocervical instrumented fusion for atlantoaxial instability in a 27-month-old child with Down syndrome: illustrative case
}

\author{
Tetsuro Ohba, MD, PhD, Kotaro Oda, MD, Nobuki Tanaka, MD, Wako Masanori, MD, PhD, Tomoka Endo, MD, and \\ Hirotaka Haro, MD, PhD
}

Department of Orthopaedic Surgery, University of Yamanashi, Yamanashi, Japan

BACKGROUND Upper cervical spine instability is one of the most serious orthopedic problems in patients with Down syndrome. Despite the recent advancement of instrumentation techniques, occipitocervical fusion remains technically challenging in the very young pediatric population with small and fragile osseous elements.

OBSERVATIONS A 27-month-old boy with Down syndrome was urgently transported to the authors' hospital because of difficulty in standing and sitting, weakness in the upper limbs, and respiratory distress. Radiographs showed os odontoideum, irreducible atlantoaxial dislocation, and substantial spinal cord compression. Emergency posterior occipitoaxial fixation was performed using $\mathrm{O}$-arm navigation. Improvement in the motor paralysis of the upper left limb was observed from the early postoperative period, but revision surgery was needed 14 days after surgery because of surgical site infection. The patient showed modest but substantial neurological improvement 1 year after the surgery.

LESSONS There are several clinical implications of the present case. It warns that Down syndrome in the very young pediatric population may lead to rapid progression of spinal cord injury and life crisis. This 27 -month-old patient represents the youngest case of atlantoaxial instability in a patient with

Down syndrome. 0-arm navigation is useful for inserting screws into very thin pedicles.

https://thejns.org/doi/abs/10.3171/CASE2175

KEYWORDS atlantoaxial instability; posterior occipitoaxial fixation; O-arm navigation; Down syndrome

Upper cervical spine instability is one of the most serious orthopedic problems in patients with Down syndrome. ${ }^{1,2}$ In particular, symptomatic patients with myelopathy from craniovertebral instability should be strongly considered as candidates for a stabilization procedure. ${ }^{3}$ Despite the recent advancement of instrumentation techniques, occipitocervical fusion $(\mathrm{OCF})$ remains technically challenging in the very young pediatric population with small and fragile osseous elements. ${ }^{4-6}$

Here, we report the successful treatment of atlantoaxial instability in a 27-month-old patient with Down syndrome via posterior occipitoaxial fixation with pedicle screws using intraoperative three-dimensional (3D) computed tomography (CT)-guided navigation with an O-arm scanner.

\section{Illustrative Case}

A 27-month-old boy with Down syndrome was urgently transported to our hospital because of difficulty in standing and sitting, weakness in the upper limbs, and respiratory distress. His height was $80.5 \mathrm{~cm}$, and his weight was $8.7 \mathrm{~kg}$. His oxygen saturation was $98 \%$ with $1 \mathrm{~L} /$ min of oxygen administration, and his left upper limb was completely paralyzed. He had poor food intake for 2 weeks before visiting our hospital and had been infused with fluids by a previous doctor.

\section{Imaging Data}

Radiography and CT showed os odontoideum and irreducible atlantoaxial dislocation (Fig. 1A and B). CT demonstrated an atlas-dens interval of $4.4 \mathrm{~mm}$ (Fig. 1B). Magnetic resonance imaging (MRI) showed substantial spinal cord compression and high T2-weighted signaling in the spinal cord (Fig. 2).

\section{Surgical Technique}

The patient was fitted with a halo vest (Lil' Angel Halo, Össur) under general anesthesia and placed prone on a Jackson table

ABBREVIATIONS 3D = three dimensional; CT = computed tomography; MRI = magnetic resonance imaging; OCF = occipitocervical fusion INCLUDE WHEN CITING Published June 7, 2021; DOI: 10.3171/CASE2175.

SUBMITTED February 3, 2021. ACCEPTED April 1, 2021.

(c) 2021 The authors, CC BY-NC-ND 4.0 (http://creativecommons.org/licenses/by-nc-nd/4.0/). 

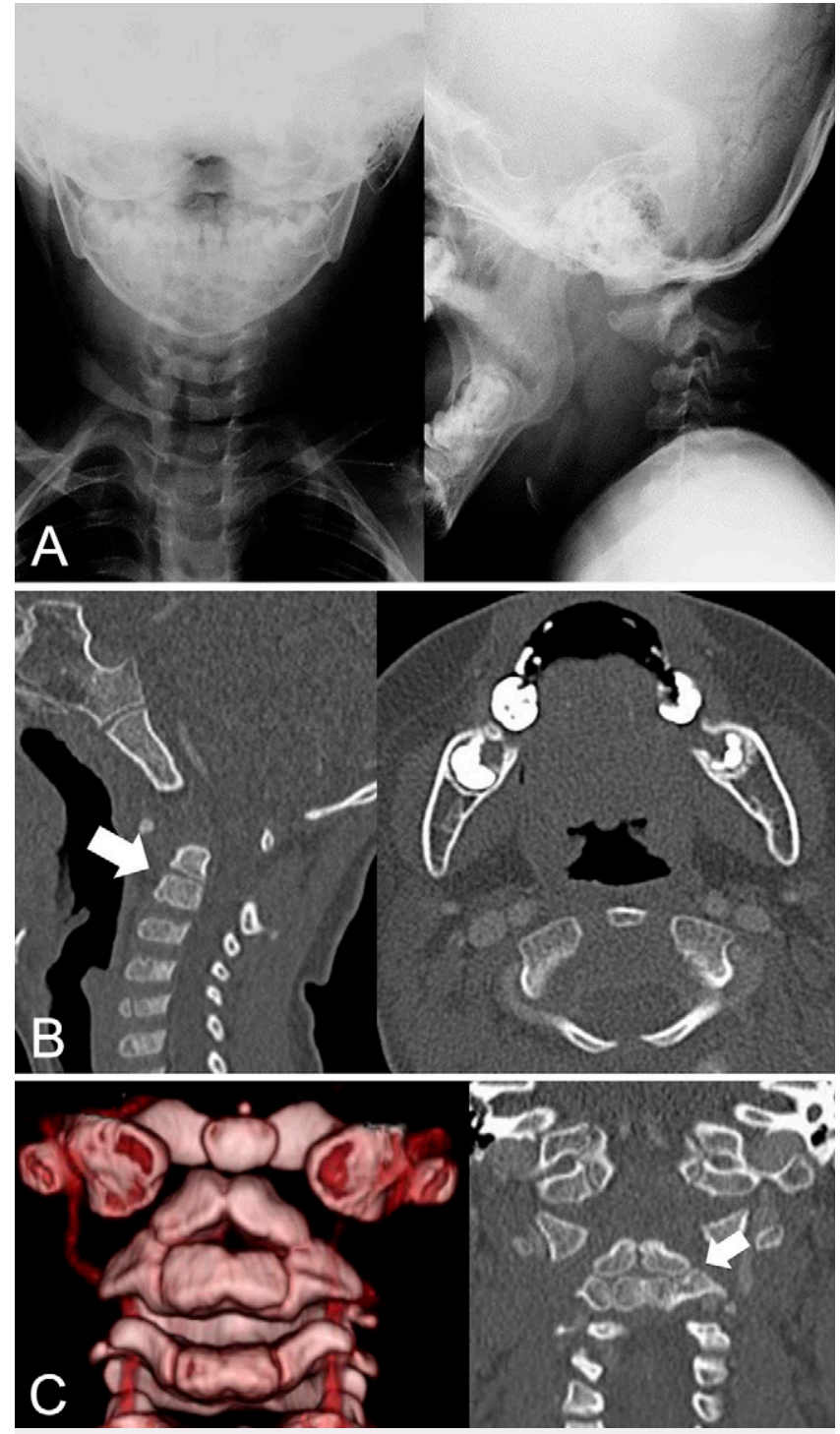

FIG. 1. Preoperative radiography (A), CT (B), and 3D model (C) demonstrated os odontoideum and atlantoaxial dislocation. The posterior arch of the atlas and odontoid process were hyperplastic. White arrows indicate the os odontoideum.

(Mizuho OSI). Ten scalp pins were inserted by probing with the fingers (Fig. 3A). Initially, we planned OCF to C3 or C4. However, his pedicles were very thin, and we could not find pedicles in the upper cervical spine into which a pedicle screw could be inserted. Additionally, we decided that the anchorage of each screw was not good because of poor bone quality, and it was necessary to extend the anchor to the level of T2 to obtain sufficient stability to support the weight of the head. Pedicle and occipital screws were placed at $\mathrm{C} 5, \mathrm{C}$, and T2 (3.5-mm diameter, 5-mm length) using intraoperative 3D CT image-guided navigation with an O-arm scanner as previously described ${ }^{7-9}$ (Fig. $3 \mathrm{~B}$ ). A plate with rods was placed (Synapse 4.0 Occipito-Cervico-Thoracic Fixation System, DePuy Synthes) along with artificial bone (Grafton, Medtronic). The halo vest was removed postoperatively with the patient under general anesthesia, and a postoperative radiographic image was obtained (Fig. 3C). The first surgery was performed urgently because of progressive respiratory dysfunction; the priority was to prevent the progression of exacerbation safely by reduction of the $\mathrm{C} 1$ ring and stabilizing $\mathrm{C} 1-2$ as much as possible. Therefore, no posterior arch resection was performed in the first operation.

\section{Postsurgical Outcome and Revision Surgery}

Improvement in the patient's motor paralysis of the upper left limb was observed from the early postoperative period, and he could swallow breast milk from the 4th day of surgery. Oxygen administration became unnecessary from the 10th day of surgery. However, 14 days after surgery, the patient had a fever of $38^{\circ} \mathrm{C}$ and a high inflammatory response in blood tests (Fig. 4A). MRI 2 weeks after surgery indicated a wound abscess and that C1-2 spinal cord compression was relieved with decreased T2-weighted signaling, but spinal cord compression was still observed (Fig. 4B). On the basis of the above information, surgical site infection was suspected, and wound debridement and posterior arch resection were performed (Fig. 4C).

The patient's fever disappeared from the 3rd day after the revision surgery, and his motor paralysis of the upper left limb improved further. The patient was able to maintain a sitting position 4 weeks after the reoperation and was discharged from the hospital. The patient showed modest but substantial neurological improvement 1 year after the surgery; he was able to maintain a standing position, shake a toy with his left hand, ingest food orally, and interact actively with his parents.

\section{Discussion}

There are several clinical implications of the present case. It warns that Down syndrome in the very young pediatric population may lead to rapid progression of spinal cord injury and life crisis. It is very rare and challenging to demonstrate cervical spine fusion in pediatric patients younger than 3 years old, and, to our knowledge, this 27-month-old patient represents the youngest case in a patient with Down syndrome. Postoperative surgical site infection and insufficient decompression with reduction of atlantoaxial instability occurred, and revision surgery was required; however, a good clinical outcome and bone fusion were observed 1 year after surgery.

Despite the reported $15 \%$ incidence of upper cervical (craniocervical and atlantoaxial) instability in Down syndrome, symptomatic upper cervical instability has been described to occur in as little as $1 \%-2 \%$ of individuals with Down syndrome. ${ }^{1,10}$ Atlantoaxial instability is a common cause of cervical pain, torticollis, and neurological dysfunction. Although very rare, the instability can rapidly lead to respiratory dysfunction and dysphagia because of spinal cord disorders, which can cause sudden death. ${ }^{1-3}$ In the present case, emergency surgery was inevitable. Radiographic studies have been conducted to evaluate the instability of the upper cervical spine in young patients with Down syndrome and to predict the appearance of future symptoms, but no consensus has yet been obtained. ${ }^{11,12}$ The present case highlights that upper cervical instability due to Down syndrome may cause rapid spinal cord injury.

A recent systematic review and meta-analysis of surgical correction of atlantoaxial instability in patients with Down syndrome indicated that a total of 149 patients with Down syndrome and atlantoaxial instability treated with upper cervical fusion have been reported. The mean age of the patients was 13.2 years, and the 

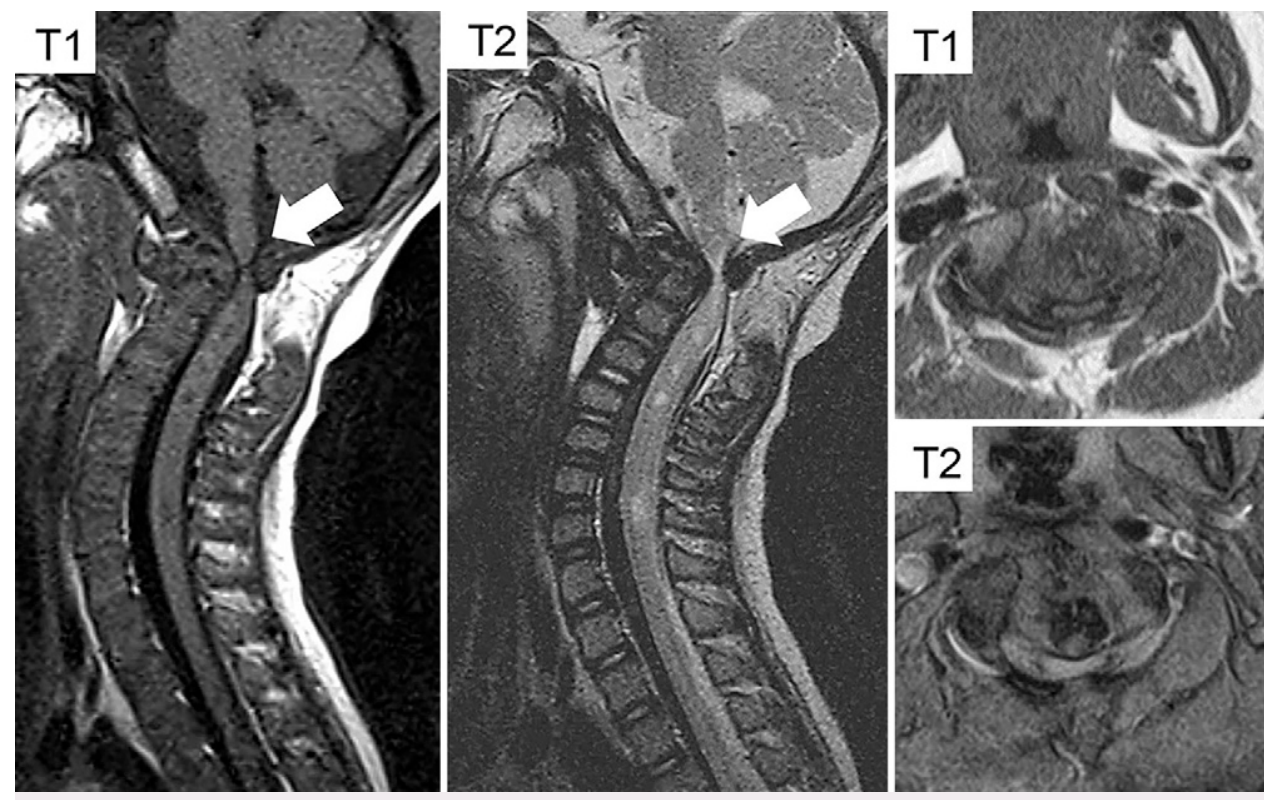

FIG. 2. MRI suggested substantial spinal cord compression (arrows) and high-intensity regions at the cervicomedullary junction on T2-weighted images.

youngest patient was 3.8 years old. ${ }^{10,13}$ Surgical treatment for upper cervical instability in skeletal dysplasia is challenging, especially in very young pediatric patients because of their small and fragile musculoskeletal structures. There are few reports of surgical treatment for upper cervical instability in patients younger than 3 years old with skeletal dysplasia due to chondrodysplasia and posttraumatic instability. ${ }^{14,15}$ The present case shows that intraoperative 3D CT image-guided navigation with an 0-arm scanner was useful for inserting screws into very thin pedicles. To our knowledge, this 27month-old boy is the youngest patient with Down syndrome to undergo posterior occipitoaxial fixation.

Orthopedic surgery for patients with Down syndrome is known to be associated with an increased risk of surgical site infection and bone union failure. ${ }^{2}$ Approximately $40 \%$ of all patients with Down syndrome who undergo cervical spine surgery have perioperative complications, and approximately $30 \%$ require revision surgery. ${ }^{1,10}$ Although revision surgery was required because of postoperative surgical site infection, substantial improvement in neurological symptoms was observed 1 year after surgery. No obvious bone fusion was found on CT 1 year after surgery, so we plan to conduct careful follow-up. The present case shows that even in a very young pediatric patient with Down syndrome, we were able to achieve good results using intraoperative 3D CT image-guided navigation with an 0-arm scanner, although perioperative complications should also be noted.

\section{Observations}

The main observations of the present case are the following. A 27-month-old boy with Down syndrome showed rapid exacerbation of neuropathy and respiratory dysfunction. Although the bone structure, including the pedicle, was very small and fragile, rigid posterior fusion could be performed using intraoperative 3D CT image-guided navigation with an 0 -arm scanner. Although revision surgery was required because of postoperative surgical site infection, substantial improvement of neurological symptoms was observed 1 year after surgery. Several cases of patients with Down syndrome and atlantoaxial instability treated with upper cervical fusion have been reported. The mean age of these patients was 13.2 years, and the youngest was 3.8 years. $^{10,13}$ Therefore, we consider that the present report is important because our 27-month-old patient is the youngest patient with atlantoaxial instability to be described to date, with the challenges of extremely small and fragile osseous elements.

\section{Lessons}

There are a few clinical implications of the present case. It warns that Down syndrome in the very young pediatric population may lead to rapid progression of spinal cord injury and life crisis. This 27-month-old patient represents the youngest case of atlantoaxial instability in a patient with Down syndrome. O-arm navigation is useful for inserting screws into very thin pedicles.

\section{References}

1. Brockmeyer D. Down syndrome and craniovertebral instability. Topic review and treatment recommendations. Pediatr Neurosurg. 1999;31(2):71-77.

2. Mik G, Gholve PA, Scher DM, et al. Down syndrome: orthopedic issues. Curr Opin Pediatr. 2008;20(1):30-36.

3. Pascual-Gallego M, Budke M, Villarejo F. Spinal stenosis at the level of atlas in a boy with Down syndrome. A case report and literature review. Article in Spanish. Neurocirugia (Astur). 2014;25(1): 29-32.

4. Ain MC, Chaichana KL, Schkrohowsky JG. Retrospective study of cervical arthrodesis in patients with various types of skeletal dysplasia. Spine (Phila Pa 1976). 2006;31(6):E169-E174.

5. Miyoshi K, Nakamura K, Haga N, Mikami Y. Surgical treatment for atlantoaxial subluxation with myelopathy in spondyloepiphyseal dysplasia congenita. Spine (Phila Pa 1976). 2004;29(21): E488-E491. 

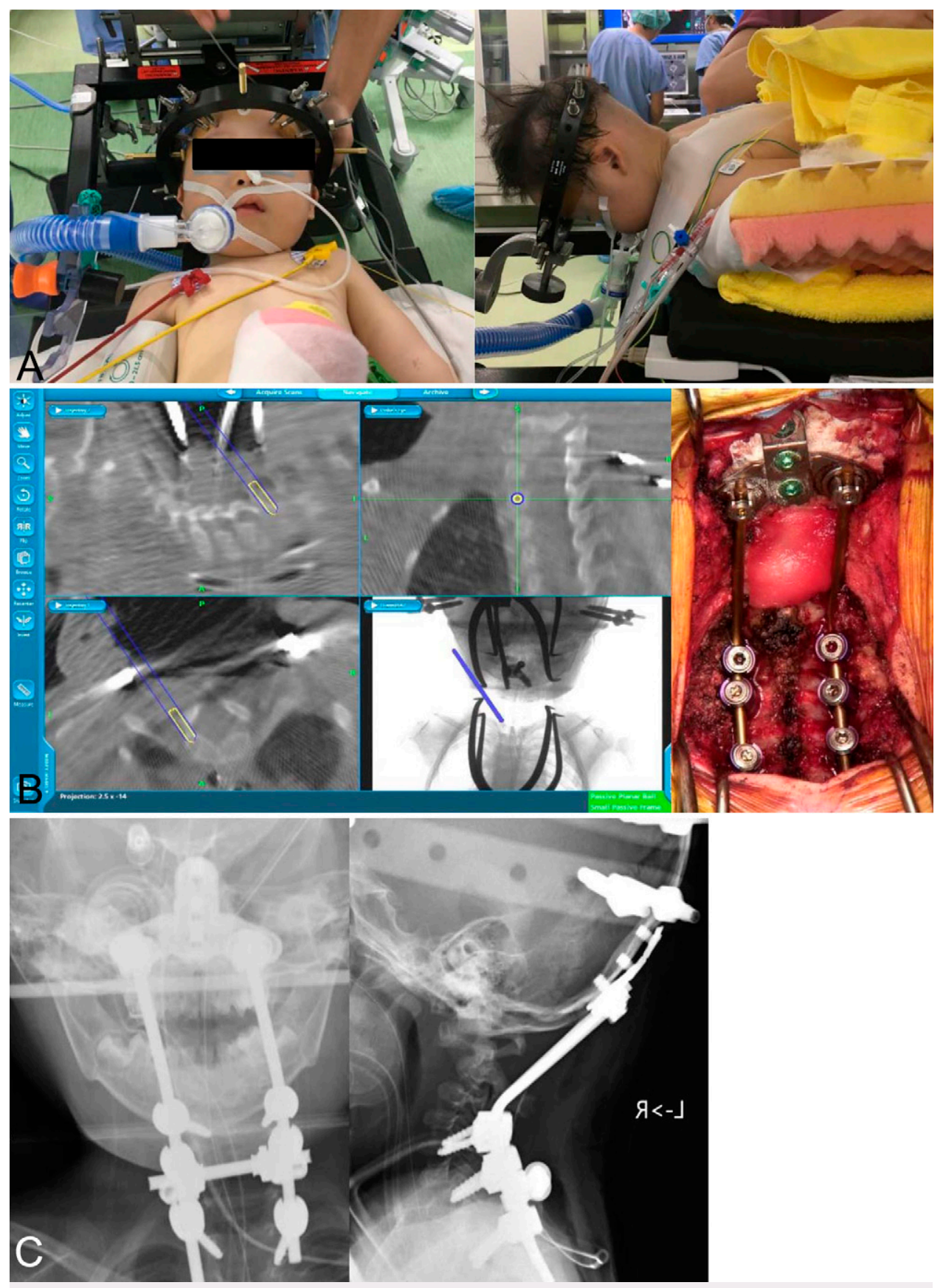

FIG. 3. The patient was fitted with a halo vest and placed prone on a Jackson table under general anesthesia. The halo vest was connected to a Jackson frame head support (Mizuho OSI) using a special radiolucent adaptor. Ten scalp pins were placed by finger palpation (A). Pedicle screws were inserted using intraoperative 3D CT image-guided navigation with an O-arm scanner (B). Postoperative radiographic images (C).

6. Haque A, Price AV, Sklar FH, et al. Screw fixation of the upper cervical spine in the pediatric population. Clinical article. J Neurosurg Pediatr. 2009;3(6):529-533.

7. Ohba T, Ebata S, Oba H, et al. Risk factors for clinically relevant loosening of percutaneous pedicle screws. Spine Surg Relat Res. 2018;3(1):79-85.

8. Ohba T, Ebata S, Fujita K, et al. Percutaneous pedicle screw placements: accuracy and rates of cranial facet joint violation using conventional fluoroscopy compared with intraoperative three-dimensional computed tomography computer navigation. Eur Spine J. 2016;25(6):1775-1780.

9. Oba H, Ebata S, Takahashi J, et al. Pedicle perforation while inserting screws using 0 -arm navigation during surgery for adolescent idiopathic scoliosis: risk factors and effect of insertion order. Spine (Phila Pa 1976). 2018;43(24): E1463-E1468. 

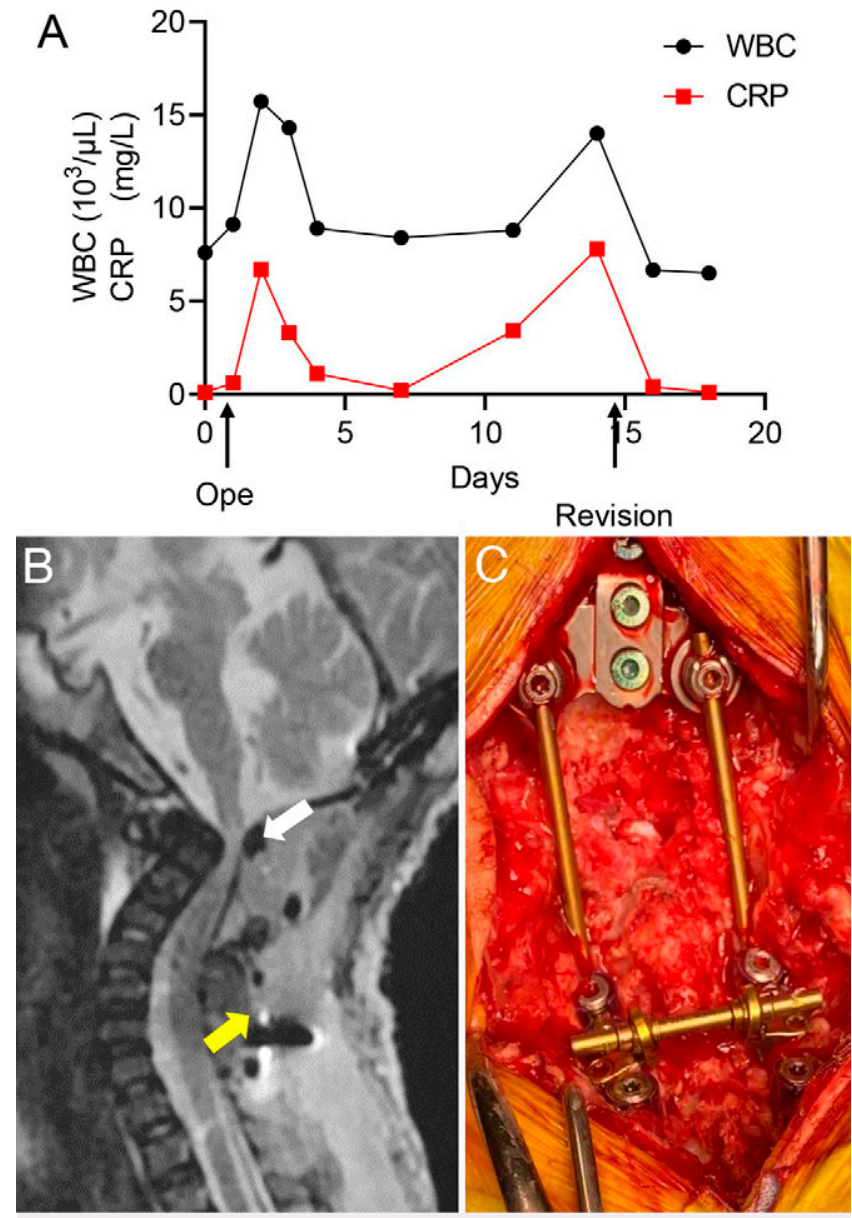

FIG. 4. Postoperative changes in inflammatory response in blood tests (A). MRI 2 weeks after surgery indicated a wound abscess (yellow arrow) and that C1-2 spinal cord compression was relieved with decreased T2-weighted signaling, but spinal cord compression (white arrow) was still observed (B). Wound debridement and posterior arch resection were performed $(\mathbf{C})$. $\mathrm{CRP}=\mathrm{C}$-reactive protein; Ope $=$ operation; $\mathrm{WBC}=$ white blood cells.
10. Yang BW, Hedequist DJ, Proctor MR, et al. Surgical fixation using screw-rod construct instrumentation for upper cervical instability in pediatric Down syndrome patients. Spine Deform. 2019;7(6): 957-961.

11. Nakamura N, Inaba $Y$, Oba M, et al. Novel 2 radiographical measurements for atlantoaxial instability in children with Down syndrome. Spine (Phila Pa 1976). 2014;39(26):E1566-E1574.

12. Watanabe K, Hirano T, Katsumi K, et al. Radiographic outcomes of upper cervical fusion for pediatric patients younger than 10 years. Spine Surg Relat Res. 2017;1(1):14-19.

13. Hofler RC, Pecoraro N, Jones GA. Outcomes of surgical correction of atlantoaxial instability in patients with Down syndrome: systematic review and meta-analysis. World Neurosurg. 2019;126: e125-e135.

14. Oba H, Takahashi J, Takano K, et al. Rigid occipitocervical instrumented fusion for atlantoaxial instability in an 18-month-old toddler with brachytelephalangic chondrodysplasia punctata: a case report Spine (Phila Pa 1976). 2017;42(23):E1380-E1385.

15. Couture D, Avery N, Brockmeyer DL. Occipitocervical instrumentation in the pediatric population using a custom loop construct: initial results and long-term follow-up experience. J Neurosurg Pediatr. 2010;5(3):285-291.

\section{Disclosures}

The authors report no conflict of interest concerning the materials or methods used in this study or the findings specified in this paper.

\section{Author Contributions}

Conception and design: Ohba. Acquisition of data: Ohba, Tanaka, Endo. Analysis and interpretation of data: Ohba, Masanori. Drafting the article: Ohba. Reviewed submitted version of manuscript: Ohba, Masanori, Haro. Approved the final version of the manuscript on behalf of all authors: Ohba. Statistical analysis: Ohba. Administrative/technical/ material support: Oda. Study supervision: Ohba, Haro.

\section{Correspondence}

Tetsuro Ohba: University of Yamanashi, Yamanashi, Japan. tooba@ yamanashi.ac.jp. 